\title{
The Atmospheric Trace Molecule Spectroscopy (ATMOS) experiment: Deployment on the ATLAS Space Shuttle missions
}

\author{
M. R. Gunson, ${ }^{1}$ M. M. Abbas, ${ }^{2}$ M. C. Abrams, ${ }^{3}$ M. Allen, ${ }^{1,4}$ L. R. Brown, ${ }^{1}$ \\ T. L. Brown, ${ }^{1}$ A. Y. Chang, ${ }^{1}$ A. Goldman, ${ }^{5}$ F. W. Irion, ${ }^{4}$ L. L. Lowes, ${ }^{1}$ E. Mahieu, ${ }^{6}$ \\ G. L. Manney, ${ }^{1}$ H. A. Michelsen, ${ }^{7}$ M. J. Newchurch, ${ }^{8}$ C. P. Rinsland, ${ }^{9}$ \\ R. J. Salawitch, ${ }^{1}$ G. P. Stiller, ${ }^{10}$ G. C. Toon, ${ }^{1}$ Y. L. Yung, ${ }^{4}$ and R. Zander ${ }^{6}$
}

\begin{abstract}
The ATMOS Fourier transform spectrometer was flown for a fourth time on the Space Shuttle as part of the ATLAS-3 instrument payload in November 1994. More than 190 sunrise and sunset occultation events provided measurements of more than 30 atmospheric trace gases at latitudes $3-49^{\circ} \mathrm{N}$ and $65-72^{\circ} \mathrm{S}$, including observations both inside and outside the Antarctic polar vortex. The instrument configuration, data retrieval methodology, and mission background are described to place in context analyses of ATMOS data presented in this issue.
\end{abstract}

\section{ATMOS Instrument}

The Atmospheric Trace MOlecule Spectroscopy (ATMOS) project was initiated in the 1970 s, when the central scientific objectives were to extend our base knowledge of the infrared spectral response of the stratosphere, measure nearly simultaneously the vertical profiles of many atmospheric constituents on a global scale, determine seasonal and annual variations in atmospheric composition, and quantify the catalytic cycles and other processes controlling ozone depletion. This goal led to the ATMOS instrument, a Fourier transform spectrometer first flown as part of the Spacelab 3 (SL-3) Space Shuttle payload in 1985. The current instrument remains essentially as described by Farmer [1987].

ATMOS was designed for operation in solar occultation mode, acquiring high-resolution, infrared solar absorption spectra of the Earth limb during orbital sunrises and sunsets. In a typical 4 minute observation period, close to 100 spectra are obtained with an apodized resolution of $\sim 0.01 \mathrm{~cm}^{-1}$. In low Earth orbit $(-300 \mathrm{~km})$ from the Space Shuttle, the height spacing between successive ATMOS spectra varies between 4 $\mathrm{km}$ to less than $1 \mathrm{~km}$ at tangent heights in the troposphere, with typical values of $\sim 2 \mathrm{~km}$ at tangent heights through the

\footnotetext{
'Jet Propulsion Laboratory, California Institute of Technology, Pasadena, CA.

${ }^{2}$ NASA Marshall Space Flight Center, Huntsville, AL.

${ }^{3}$ SAIC, NASA Langley Research Center, Hampton, VA.

${ }^{4}$ California Institute of Technology, Pasadena, CA.

${ }^{5}$ Department of Physics, University of Denver, Denver, CO.

${ }^{6}$ Institute of Astrophysics, Univ. of Liège, Liège-Cointe, Belgium.

${ }^{7}$ Harvard University, Cambridge, MA.

${ }^{8}$ University of Alabama in Huntsville, Huntsville, AL.

${ }^{9}$ NASA Langley Research Center, Hampton, VA.

${ }^{10}$ IMK, Forschungszentrum Karlsruhe, Karlsruhe, Germany.
}

Copyright 1996 by the American Geophysical Union.

Paper number 96GL01569

0094-8534/96/96GL-01569\$05.00 stratosphere. The projection of the instrument field-of-view (1 $-2.8 \mathrm{mrad}$ ) at the tangent point limits the effective vertical resolution in retrieved profiles to $\sim 4 \mathrm{~km}$. The signal-to-noise ratios of the resulting spectra are in the range 250 to $100: 1$, depending on the optical bandpass filter used to select spectral regions between $600-4750 \mathrm{~cm}^{-1}$ (16.6 - 2.1 microns). Up to eight different optical filters were carried on each flight, with some changed between flights to extend the multiple species measurement within individual observations, while preserving the signal-to-noise ratio of the resulting spectra. Abrams et al. [this issue (a)] discuss the estimated precision reported with the ATMOS vertical profiles and compare these with the standard deviation of the mean of several profiles obtained at low latitudes. The altitude range over which ATMOS profiles are reported, together with a general guide to the precisions achieved for each gas are shown in Figure 1. The upper altitudes coincide with the first appearance of absorption features of a particular gas that vary slightly between successive occultations. The lower altitude limit is determined by a combination of factors: rapid increase in opacity through the troposphere, aerosols and cloud tops obscuring the sun, lack of accuracy in assigning tangent heights and pressures to individual spectra, and the difficulty in retrieving stratospheric constituent volume mixing ratios (vmrs) below their peak concentrations. Issues limiting the lower altitude range will be addressed in future analyses.

The data processing and inversion methods employed with the ATMOS data follow those described by Norton and Rinsland [1991]. As some changes have been incorporated to enhance routine data processing and to distinguish between this current dataset and previous published analyses, the ATMOS data utilized in the papers in this issue have been ascribed to 'Version 2'. In brief, after transformation of the interferograms to power spectra, an average exoatmospheric spectrum is formed for each occultation against which ratios are made with successive atmospheric spectra. The resulting atmospheric transmission spectra are essentially free of solar and instrumental spectral features. The inversion of these transmission spectra follows a general sequence: assignment of a first-guess tangent height based on spacecraft ephemeris data, refinement of the tangent height (or more correctly, tangent pressure) from spectral fitting to the observed $\mathrm{CO}_{2}$ absorption bands [Abrams et al., 1996], and retrieval of atmospheric temperature-pressure profiles [Stiller et al., 1995], followed by a final tangent pressure assignment. Retrievals of vmr profiles for individual species use spectral intervals that are chosen based on the shape and strength of target gas absorption, the insensitivity of these features to errors in temperature, and their relative freedom from interfering absorption from other constituents [e.g., Gunson 
et al., 1990]. The ATMOS spectral linelist and associated spectroscopic uncertainties (leading to systematic uncertainties in the vmrs) are discussed in Brown et al. [1996].

\section{ATMOS and ATLAS}

The results from the 1985 SL-3 deployment have been extensively reported in the literature, and have demonstrated the potential for high-resolution, infrared solar occultation observations to measure the vertical profiles of more than thirty atmospheric trace gases. Included in this dataset were measurements of vertical profiles of trace gases in the stratosphere not measured previously [e.g., $\mathrm{N}_{2} \mathrm{O}_{5}$, Toon et al., 1986; $\mathrm{ClONO}_{2}$, Zander et al., 1986; $\mathrm{HO}_{2} \mathrm{NO}_{2}$, Rinsland et al., 1986a; $\mathrm{CH}_{3} \mathrm{Cl}$, Park et al., 1986; $\mathrm{COF}_{2}$, Rinsland et al., $1986 \mathrm{~b}, \mathrm{SF}_{6}$, Rinsland et al., 1990], which, combined with the measured vertical profiles of other trace constituents, provided opportunities to critically evaluate aspects of our understanding of the photochemistry of the upper stratosphere [e.g., McElroy and Salawitch, 1989; Allen and Delitsky, 1990; Natarajan and Callis, 1991]. The absorption features of several gases were discernible well above heights in the stratosphere, providing information for analyses on the composition of the mesosphere and thermosphere [Kumar et al., 1995; Lopez-Puertas et al., 1992].

The operational success of ATMOS was realized over the three flights of the 1992-1994 ATLAS series (AT-1, AT-2, and AT-3). Together, these missions provided observations of vertical profiles of atmospheric constituents throughout the tropics and mid-latitudes, in both hemispheres and over two seasons (Figure 2). In addition, ATMOS obtained measurements inside and outside the Arctic polar vortex during AT-2, and sampled inside and outside the Antarctic vortex in AT-3.

The early November mission of AT-3 provided the first opportunity to obtain space-based measurements on the chemical and dynamical state of the Antarctic vortex with the breadth of species and measurement precision provided by
ATMOS, comparing conditions inside and outside the region of maximum ozone loss that had occurred in the preceding months. Manney et al. [this issue] discuss meteorological conditions relevant to ATMOS observations during AT-3. Rinsland et al. [this issue $(\mathrm{a}, \mathrm{b})$ ] have used the ability of ATMOS to measure the components of the family of reactive nitrogen species as well as $\mathrm{H}_{2} \mathrm{O}$ and $\mathrm{CH}_{4}$, to show that the level of denitrification within the vortex through formation and sedimentation of polar stratospheric clouds (PSCs) occurs at a slightly higher altitude of $\sim 20 \mathrm{~km}$ than the maximum level of dehydration (as measured by $\mathrm{H}_{2} \mathrm{O}+$ $2 \times \mathrm{CH}_{4}$ ), which appears at $\sim 18 \mathrm{~km}$ altitudes. Near $25 \mathrm{~km}$, the long-lived tracers $\mathrm{N}_{2} \mathrm{O}$ and $\mathrm{CH}_{4}$ are measured with vmrs equivalent to those found at $\sim 50 \mathrm{~km}$ [Abrams et al., this issue (b)]. This air brings higher levels of inorganic chlorine $(\mathrm{HCl}$ $+\mathrm{ClONO}_{2}+\mathrm{ClO}$ ), which, by the time of the AT-3 mission, has been converted mainly to $\mathrm{HCl}$ under the low ozone levels in the vortex, which lead also to higher vmrs of NO [Rinsland et al., this issue (a)]. In contrast, measurements obtained by ATMOS during AT-2 through the Arctic vortex in April 1993 show similar descent rates but, because of the shorter descent period, less total descent [Abrams et al., this issue (c)].

The conditions within the polar vortices can be viewed as perturbed relative to the typical conditions encountered at mid-latitudes or within the tropics. However, detailed model studies of mid-latitude ATMOS observations of chlorine sink and reservoir species reveal a deficit in the computed levels of $\mathrm{HCl}$ that can be reconciled if an additional source of stratospheric $\mathrm{HCl}$ production, such as $\mathrm{ClO}+\mathrm{OH} \rightarrow \mathrm{HCl}+\mathrm{O}_{2}$, is allowed [Michelsen et al., this issue]. The internal consistency of the ATMOS halogen measurements has been evaluated previously by Zander et al. [1992] by comparing the altitude variation of the total chlorine-atom content from all measured species, which includes not only the major sink and reservoirs, but also most of the major source gases. This analysis is extended using the AT-3 measurements from ATMOS [Zander et al., this issue (a)] and MAS (Millimeter

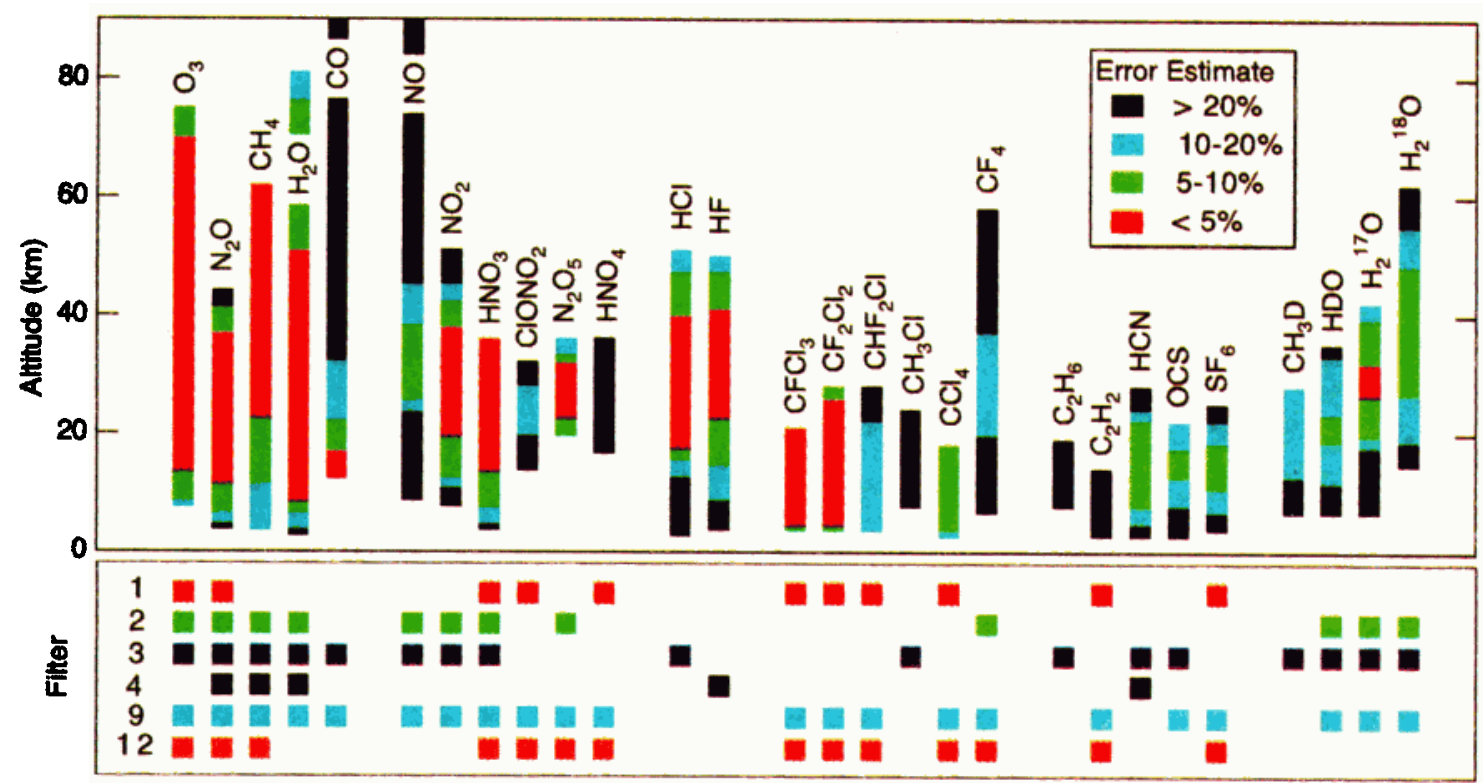

Figure 1. Vertical range of trace gas measurement in ATMOS 'Version 2' data. Shadings correspond to estimated $1 \sigma$ precision error in retrieved volume mixing ratio The lower panel maps gases to the ATMOS optical bandpass filters in which they are measured. The spectral bandpasses (in $\mathrm{cm}^{-1}$ ) correspond to: filter 1, 600-1180; filter 2, 1100-2000; filter 3, 1580-3340; filter 4, 3150-4800; filter 9, 600-2450; filter 12, 600-1400. 


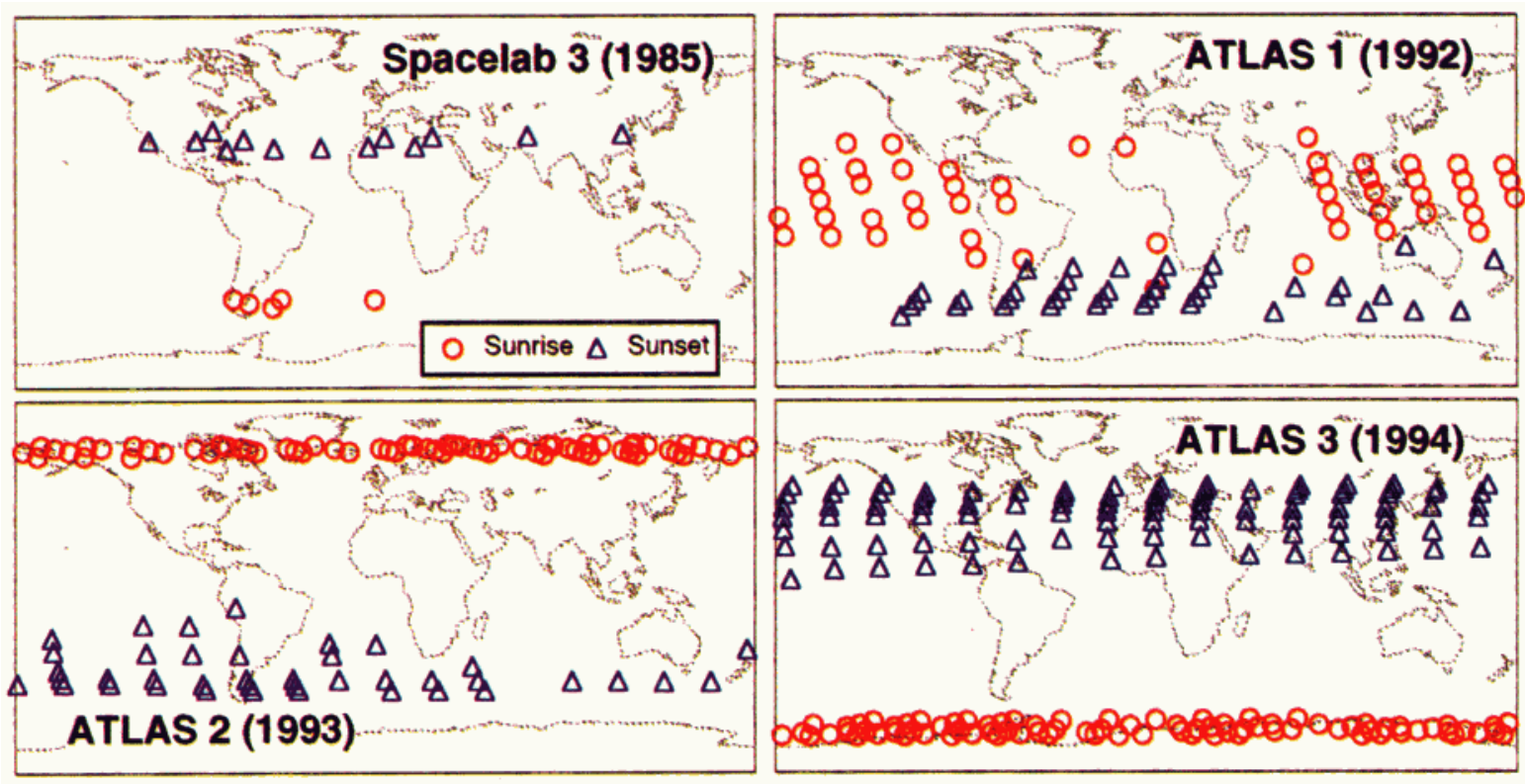

Figure 2. Sunrise (circles) and sunset (triangles) occultations observed by ATMOS on Space Shuttle for (a) Spacelab 3, 29 April-May 7, 1985; (b) ATLAS-1, 24 March - 3 April 1992; (c) ATLAS-2, 8 - 16 April 1993; and (d) ATLAS-3, 3 - 14 Nov 1994.

Atmospheric Sounder) which measures ClO [Aellig et al., this issue], and the total stratospheric chlorine-loading compared with values measured by ATMOS on all four shuttle flights. This shows that the measured trends in stratospheric inorganic chlorine and fluorine are consistent with the growth in halocarbon source gases, such as chlorofluorocarbons. Following the approach discussed in Gunson et al. [1994], the measured upper stratospheric $\mathrm{HCl}$ and $\mathrm{HF}$ from all four shuttle flights are shown in Figure 3. The roughly 9.5 year interval between the first and latest deployment is sufficiently long to allow significant trends to be discernible not only in total chlorine and fluorine loading, but also in many individual species [Rinsland et al., this issue (c); Zander et al., this issue (b)].

The broad range of species measured at the precision and accuracy achieved by ATMOS over a long time base of observations provides correlative measurements with satellite instruments such as those on the Upper Atmospheric Research Satellite [see special issue, J. Geophys. Res., 101, No. D6, 1996]. Relating broad but global space-based observations to the detailed measurements made by in situ sensors carried on research aircraft has not always been possible. Chang et $a l$. [this issue, $(a, b)$ ] explore inter-comparisons of ATMOS measurements at northern mid-latitudes with those made by instruments on the ER-2 deployed in the same region. These comparisons reveal good agreement for many species to within a few percent, and certainly within the estimated precision of either data set, confirming the potential to utilize observations from such disparate sources to study atmospheric processes. However larger systematic differences were evident in the measurements of CO [Chang et al., this issue (a)], $\mathrm{CCl}_{4}$, and $\mathrm{HCl}$ [Chang et al., this issue (b)]. The good agreement for measurements of $\mathrm{NO}_{y}$ (in correlations against $\mathrm{N}_{2} \mathrm{O}$ ) corroborates the accuracy of the in situ measurements obtained with a gold catalyst total- $\mathrm{NO}_{\mathrm{y}}$ sensor. Newchurch et al. [this issue] describe the sensitivities and results of accounting for the rapid diurnal variation of NO and $\mathrm{NO}_{2}$ along the line-of-sight through the terminator. The excellent agreement of the diurnally corrected NO from
ATMOS with in situ measurements on the ER-2 provides evidence that these space-borne measurements can be used to quantify $\mathrm{NO}_{\mathbf{x}}$ in the lower stratosphere.

Co-located measurements of water and methane by ATMOS have revealed further evidence of the seasonal variation in the vmr of water entering the stratosphere [Abbas et al., this issue, (a)]. Also, the sum $\mathrm{H}_{2} \mathrm{O}+2 \times \mathrm{CH}_{4}$ has been shown to be relatively constant in the lower stratosphere but exhibits a maximum between 35 and $65 \mathrm{~km}$, consistent with oxidation
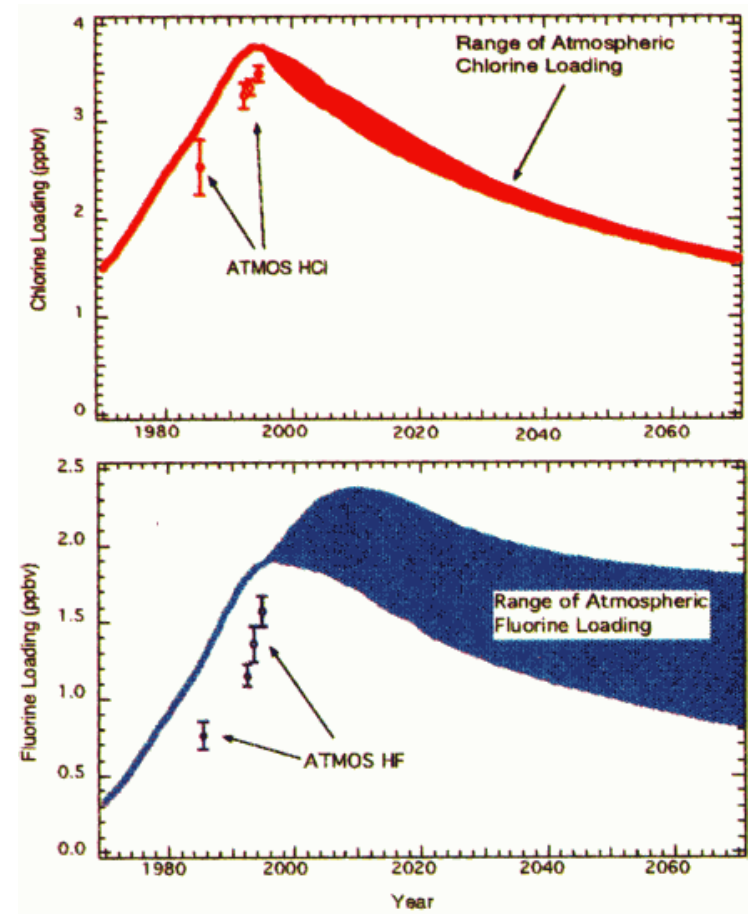

Figure 3. Modeled changes in total tropospheric chlorine $\left(\mathrm{Cl}_{\mathrm{y}}\right)$ and fluorine $\left(\mathrm{F}_{\mathrm{y}}\right)$ as described by Gunson et al. [1994] (Fig. 3), along with ATMOS data for upper stratospheric $\mathrm{HCl}$ and $H F$ from 1985, 1992, 1993, and 1994. 
of $\mathrm{H}_{2}$ as a net source of water [Abbas et al., this issue, (b)]. The ATMOS spectral range and resolution allow analyses of several isotopomers of ozone, water, and methane, and offers the potential for new insight into atmospheric photochemical and dynamical processes. Irion et al. [this issue, (a)] investigate the vertical structure of heavy ozone and find enrichment of ${ }^{16} \mathrm{O}^{16} \mathrm{O}^{18} \mathrm{O}$ and ${ }^{16} \mathrm{O}^{18} \mathrm{O}^{16} \mathrm{O}$ to be relatively constant with altitude and season. Irion et al. [this issue, (b)] quantitatively investigate the oxidation of $\mathrm{CH}_{3} \mathrm{D}$ as a stratospheric source of HDO. Moyer et al. [this issue] use ATMOS measurements of deuterium depletion in stratospheric water as evidence that much of troposphericstratospheric exchange is associated with rapid convective events in the troposphere.

\section{ATMOS Data}

The ATMOS 'Version 2' data are available through an anonymous ftp-site at ftp://remus.jpl.nasa.gov/pub/atmos/

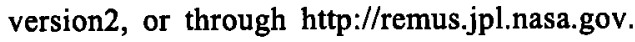

Acknowledgments. This work was carried out at the Jet Propulsion Laboratory, California Institute of Technology, under contract to the National Aeronautics and Space Administration. The authors wish to thank the many people who have contributed their time and effort to the success of ATMOS including Reinhard Beer, Barney Farmer, Julie Foster, Greg Goodson, Gindi Lynch, Bert McKenna, Fred O'Callaghan, Odell Raper, Rudy Schindler, Bhaswar Sen to name but a few.

\section{References}

Abbas, M. M., et al., Seasonal variations of water vapor in the lower stratosphere inferred from ATMOS/ATLAS-3 measurements of $\mathrm{H}_{2} \mathrm{O}$ and $\mathrm{CH}_{4}$, Geophys. Res. Lett., this issue, 1996a.

Abbas, M. M., et al., The hydrogen budget of the stratospherc inferred from ATMOS measurements of $\mathrm{H}_{2} \mathrm{O}$ and $\mathrm{CH}_{4}$, Geophys. Res. Lett., this issue, 1996b.

Abrams, M. C., et al., Pressure sounding of the middle atmosphere from ATMOS solar occultation measurements of atmospheric $\mathrm{CO}_{2}$ absorption lines, Appl. Opt., in press, 1996.

Abrains, M. C., et al., On the assessment and uncertainty of atmospheric trace gas burden measurements with high resolution infrared solar occultation spectra from space, Geophys. Res. Lett., this issue, 1996a.

Abrams, M. C., et al., ATMOS/ATLAS-3 observations of descent in the Antarctic vortex in November 1994, Geophys. Res. Lett., this issue, 1996b.

Abrams, M. C., et al., Trace gas transport in the Arctic vortex inferred from ATMOS ATLAS-2 observations during April 1993, Geophys. Res. Lett., this issue, 1996c.

Aellig, C. P., et al., Latitudinal distribution of upper stratospheric $\mathrm{ClO}$ as derived from space borne microwave spectroscopy, Geophys. Res. Lett., this issue, 1996.

Allen, M., and M. L. Delitsky, Stratospheric NO, $\mathrm{NO}_{2}$, and $\mathrm{N}_{2} \mathrm{O}_{5}$ : A comparison of model results with Spacelab 3 Atmospheric Trace Molecule Spectroscopy (ATMOS) measurements, J. Geophys. Res., 95, 14077-14082, 1990.

Brown, L. R., et al., The 1995 Atmospheric Trace Molecule Spectroscopy (ATMOS) linelist, Appl. Opt., in press, 1996.

Chang, A. Y., et al., A comparison of measurements from ATMOS and instruments aboard the ER-2 aircraft: Tracers of atmospheric transport, Geophys. Res. Lett., this issue, 1996a.

Chang, A. Y., et al., A comparison of measurements from ATMOS and instruments aboard the ER-2 aircraft: Halogenated gases, Geophys. Res. Lett., this issue, 1996b.

Farmer, C. B., High resolution infrared spectroscopy of the Sun and the Earth's atmosphere from space, Mikrochim. Acta (Wien), III, 189$214,1987$.

Gunson, M. R., et al., Measurements of $\mathrm{CH}_{4}, \mathrm{~N}_{2} \mathrm{O}, \mathrm{CO}, \mathrm{H}_{2} \mathrm{O}$, and $\mathrm{O}_{3}$ in the middle atmosphere by the ATMOS experiment on Spacelab $3, J$. Geophys. Res., 95, 13867-13882, 1990.

Gunson, M. R., et al., Increase in levels of stratospheric chlorine and fluorine loading between 1985 and 1992, Geophys. Res. Lett., 21, 2223-2226, 1994.

Irion, F. W., et al., Heavy ozone enrichments from ATMOS infrared solar spectra, Geophys. Res. Lett., this issue, 1996a.
Irion, F. W., et al., Stratospheric observations of $\mathrm{CH}_{3} \mathrm{D}$ and HDO from ATMOS infrared solar spectra: Enrichments of deuterium in methane and implications for HD, Geophys. Res. Lett., this issue, 1996b.

Kumar, C. K., et al., ATMOS/ATLAS-1 measurements of thermospheric and mesospheric nitric oxide, J. Geophys. Res., 100, 16839-16846, 1995.

Lopez-Puertas, M., et al., Analysis of the upper atmosphere $\mathrm{CO}_{2}\left(\mathrm{v}_{2}\right)$ vibrational temperatures from ATMOS/Spacelab 3 observations, $J$. Geophys. Res., 97, 20469-20478, 1992.

Manney, G. L., R. Swinbank, and A. O'Neill, Stratospheric meteorological conditions for the 3-12 Nov 1994 ATMOS/ATLAS-3 measurements, Geophys. Res. Lett., this issue, 1996.

McElroy, M. B., and R. J. Salawitch, Changing composition of the global stratosphere, Science, 243, 763-770, 1989.

Michelsen, H. A., et al., Stratospheric chlorine partitioning: Constraints from shuttle-borne measurements of $[\mathrm{HCl}],\left[\mathrm{ClNO}_{3}\right]$, and $[\mathrm{ClO}]$, Geophys. Res. Lett., this issue, 1996.

Moyer, E. J., et al., ATMOS stratospheric deuterated water and implications for troposphere-stratosphere transport, Geophys. Res. Lett., this issue, 1996.

Natarajan, M., and L. B. Callis, Stratospheric photochemical studies with Atmospheric Trace Molecule Spectroscopy (ATMOS) measurements, J. Geophys. Res., 96, 9361-9370, 1991.

Newchurch, M. J., et al., Stratospheric NO and $\mathrm{NO}_{2}$ abundances from ATMOS solar-occultation measurements, Geophys. Res. Lett., this issue, 1996.

Norton, R. H., and C. P. Rinsland, ATMOS data processing and science analysis methods, Appl. Opt., 30, 389-400, 1991.

Park, J. H., et al., Spectroscopic detection of $\mathrm{CH}_{3} \mathrm{Cl}$ in the upper troposphere and lower stratosphere, Geophys. Res. Lett., 13, 765$768,1986$.

Rinsland, C. P., et al., Evidence for the presence of the $802.7 \mathrm{~cm}^{-1}$ band $Q$ branch of $\mathrm{HO}_{2} \mathrm{NO}_{2}$ in high resolution solar absorption spectra of the stratosphere, Geophys. Res. Lett., 13, 761-764, 1986a.

Rinsland, C. P., et al., Detection of carbonyl fluoride in the stratosphere, Geophys. Res. Lett., 13, 769-772, 1986b.

Rinsland, C. P., L. R. Brown, and C. B. Farmer, Infrared spectroscopic detection of sulfur hexafluoride $\left(\mathrm{SF}_{6}\right)$ in the lower stratosphere and upper troposphere, J. Geophys. Res., 95, 5577 - 5585, 1990.

Rinsland, C. P., et al., ATMOS/ATLAS-3 measurements of stratospheric chlorine and reactive nitrogen partitioning inside and outside the November 1994 Antarctic vortex, Geophys. Res. Lett., this issue, 1996a.

Rinsland, C. P., et al., ATMOS measurements of $\mathrm{H}_{2} \mathrm{O}+2 \mathrm{CH}_{4}$ and total reactive nitrogen in the November 1994 Antarctic stratosphere: Dehydration and denitrification in the vortex, Geophys. Res. Lett., this issue, 1996b.

Rinsland, C. P., et al., Trends of OCS, $\mathrm{HCN}, \mathrm{SF}_{6}, \mathrm{CHClF}_{2}$ (HCFC-22) in the lower stratosphere from 1985 and 1994 Atmospheric Trace Molecule Spectroscopy experiment measurements near $30 \mathrm{~N}$ latitude, Geophys. Res. Lett., this issue, 1996c.

Stiller, G. P., et al., Stratospheric and mesospheric pressure-temperature profiles from the rotational analysis of $\mathrm{CO}_{2}$ Lines of ATMOS/ATLAS-1 Observations, J. Geophys. Res., 100, 3107-3117, 1995.

Toon, G. C., C. B. Farmer, and R. H. Norton, Detection of stratospheric $\mathrm{N}_{2} \mathrm{O}_{5}$ by infrared remote sounding, Nature, 319, 570-571, 1986.

Zander, R., et al., Observation of Several Chlorine Nitrate (ClONO Bands in Stratospheric Infrared Spectra, Geophys. Res. Lett., 13, 757-760, 1986.

Zander, R., et al., The 1985 Chlorine and fluorine inventories in the stratosphere based on ATMOS observations at 30 North latitude, $J$. Atm. Chem., 15, 171-186, 1992.

Zander, R., et al., The 1994 northern midlatitude budget of stratospheric chlorine derived from ATMOS/ATLAS-3 observations, Geophys. Res. Lett., this issue, 1996a.

Zander, R., et al., Increase of stratospheric carbon tetrafluoride $\left(\mathrm{CF}_{4}\right)$ based on ATMOS observations from space, Geophys. Res. Lett., this issue, 1996b.

M. R. Gunson, Jet Propulsion Laboratory, 4800 Oak Grove Drive, Mail-Stop 183-301, Pasadena, CA 91109 (email: Michael.R.Gunson@ jpl.nasa.gov).

(Received April 3, 1996; revised April 25, 1996; accepted May 2, 1996.) 